\title{
Activation of the signal transducer and activator of transcription 1 signaling pathway in thymocytes from HIV-1-infected human thymus
}

\author{
Eric D. Miller, Jonathan A. Smith, Monika Lichtinger, Liping Wang, and Lishan Su \\ Lineberger Comprehensive Cancer Center, Department of Microbiology and Immunology, The \\ University of North Carolina at Chapel Hill, Chapel Hill, NC 27599-7295, USA
}

\begin{abstract}
Objective-To identify HIV-induced host factors in the severe combined immunodeficient (SCID)-hu Thy/Liv mouse that may contribute to HIV pathogenesis in the thymus.

Design-To identify genes specifically altered by HIV-1 infection using the cDNA microarray assay, SCID-hu Thy/Liv organs derived from the same donors were used. Therefore, no genetic variations existed between HIV and mock-infected samples. In addition, the 12-14 day postinfection timepoint was chosen because no significant thymocyte depletion was detected in HIVinfected Thy/Liv organs, so mRNA from the same cell types could be compared.
\end{abstract}

Methods-Using SCID-hu Thy/Liv mice constructed from the same donor tissues, we analysed the expression of 9183 host genes in response to HIV infection with cDNA microarrays. Expression of selected genes with more than threefold induction was confirmed by measuring RNA (reverse transcriptase-polymerase chain reaction; RT-PCR) and proteins.

Results-HIV-1 (JD or NL4-3) infection of the SCID-hu Thy/Liv mouse led to more than threefold induction of 19 genes, 12 of which were IFN-inducible and six were unknown EST clones. We confirmed induction by RT-PCR and protein blots. Both signal transducer and activator of transcription (STAT) 1 and STAT2 proteins were induced, and STAT1 was also activated by phosphorylation at the Tyr701 and Ser727 sites in human thymus infected with HIVJD or NL4-3. Treatment of human fetal thymus organ culture or human thymocytes with recombinant HIV-1 gp120 proteins also led to induction or activation of STAT1.

Conclusion-HIV-1 infection of the thymus led to activation of the STAT1 signaling pathway in thymocytes, which may contribute to HIV-1 pathogenesis in the thymus.

\section{Keywords}

HIV/AIDS; human fetal thymus organ culture; microarray; severe combined immunodeficient-hu Thy/Liv; signal transducer and activator of transcription 1; thymus 


\section{Introduction}

The thymus is an early site of HIV-1 replication [1], and thymic organs from HIV-1-infected patients show profound parenchymal damage and involution [2]. Moreover, HIV-1-induced thymus dysfunction has been correlated with faster AIDS progression in pediatric patients [3]. As the thymus organ is difficult to study in humans, a small animal model for the analysis of human thymopoiesis [severe combined immunodeficient (SCID)-hu Thy/Liv mouse] has been constructed by engrafting fragments of human fetal liver and thymus into the immunodeficient C.B-17 scid/scid (SCID) mouse [4]. After inoculation of the SCID-hu Thy/Liv mouse with HIV-1, replication of pathogenic HIV-1 isolates reaches high levels at 2 weeks post-infection, followed by depletion of CD4 thymocytes between 3 and 6 weeks post-infection [5-8]. A higher rate of replication and thymocyte depletion is observed with rapidly replicating, syncytium-inducing virus isolated from AIDS patients than with slowly replicating, non-syncytium-inducing virus isolated from the same patients before the development of AIDS, or from long-term non-progressor patients [9]. Unlike in tissue cultures, mutations in HIV accessory genes significantly attenuated the replication and pathogenesis of HIV-1 in SCID-hu Thy/Liv mice [10,11]. The SCID-hu Thy/Liv mouse thus provides a relevant in vivo model to evaluate HIV-1 replication and pathogenicity in the human thymus.

Both indirect and direct mechanisms of thymocyte depletion have been implicated in HIV-1infected thymus organs $[5,8,12]$. High levels of MHC class I are induced on all immature thymocytes, but only a small fraction of them are directly infected by HIV-1 [13]. Apoptosis has been associated with HIV-1-induced T cell death both in vitro and in vivo [14,15]. In the Thy/Liv organ, thymocytes with condensed nuclei were detected in HIV-1-infected Thy/Liv organs by thin section light microscopy and by electron microscopy [5]. Biochemically, partial chromosomal loss [5] and DNA strand breaks [8] are associated with HIV-1-induced thymocyte depletion. Consistently, it has recently been reported that indirect mechanisms of thymocyte depletion are primarily involved in the thymus of SIV-infected monkeys [16]. In support of the direct lytic infection mechanism, thymocyte depletion may be achieved by a number of HIV-1-encoded factors with cytotoxic or cytostatic activities, as demonstrated in T cells in vitro. High viral loads during the early stages of HIV-1-induced thymocyte depletion in the SCID-hu Thy/Liv mouse have been proposed to lead to direct cytolytic infection and thymocyte depletion [12]. In addition, the intrathymic T progenitor cells can be directly infected and depleted to lead to thymocyte depletion by blocking $\mathrm{T}$ cell development [8].

The thymus microenvironment is essential for T cell development. Destruction of thymic epithelial cells and the induction of various cytokines have been reported in the human thymus and in the SCID-hu Thy/Liv mouse after HIV-1 infection [2,7,13,17]. This may impair $\mathrm{T}$ cell development and result in thymocyte depletion. Indeed, two recent reports $[18,19]$ showed that hematopoietic progenitor cells in HIV-1-infected Thy/Liv organs are preferentially depleted or suppressed by indirect mechanisms, before thymocyte depletion. Destruction of the thymus organ by HIV-1 infection is probably caused by the induced expression of viral and host pathogenic factors. Identification of the host genes will help our understanding of HIV pathogenesis in the thymus. The complementary DNA microarray 
assay $[20,21]$ has recently been developed to analyse differential gene expression profiles at the genome level. Using the cDNA microarray assay and confirmed by reverse transcriptase-polymerase chain reaction (RT-PCR) or Western blot assays, we showed that HIV-1 infection of the thymus organ led to enhanced expression of a number of genes involved in the Stat1 signaling pathway. We demonstrated that HIV-1 infection led to activation of the signal transducer and activator of transcription (STAT) 1 proteins. We further showed that incubation of human fetal thymus organ culture (HF-TOC) or thymocytes with recombinant HIV-1 gp120 led to the induction or activation of the STAT1 protein.

\section{Materials and methods}

\section{Reagents}

Monoclonal antibodies reactive with human CD4 and CD8 cells were purchased from Becton Dickinson (San Jose, CA, USA). Anti-MHCI monoclonal antibody (mAb) W6/32 was from Biodesign (Kennebunk, ME, USA). Polyclonal anti-STAT1, anti-P-STAT1-Ser (or Tyr) and anti-STAT2 antibodies were purchased from New England Biolabs (Beverly, MA, USA). The monoclonal anti-p91 (STAT1) antibody was from Santa Cruz Biotech (Santa Cruz, CA, USA). The HIV-1 isolates (JD and NL4-3) used in this study have been described previously $[8,22]$. JD used both CXCR4 and CCR5 and NL4-3 used exclusively CXCR4 as co-receptors for entry in vitro. SDF1a and IFN $\gamma$ (R\&D Systems, Minneapolis, MN, USA) were used as recommended. Recombinant gp120 proteins (from X4-tropic IIIB or R5-tropic BaL isolates) were kindly provided by W. Chen (GlaxoSmithKline, Research Triangle Park, NC, USA).

\section{Infection of severe combined immunodeficient-hu Thy/Liv mice}

Animal transplantation and HIV infection procedures of SCID-hu Thy/Liv mice have been described previously $[8,22]$. Briefly, SCID-hu Thy/Liv mice were infected with supernatant collected from phytohemaglutinin-activated peripheral blood mononuclear cells (PBMC) containing no HIV-1 (mock) or $4 \times 10^{4}$ to $10^{5} \mathrm{IU} / \mathrm{ml}$ of HIV-1 (JD or NL4-3). The Thy/Liv organs were harvested at the times indicated and thymocyte sub-populations were analysed by FACS (CD4-PE, CD8-TC, MHCI-FITC), and cell-associated p24 production (pg/10 thymocytes) was measured using a p24 enzymelinked immunosorbent assay kit [13].

\section{HIV infection or treatment of human fetal thymus organ culture or thymocytes with recombinant HIV-1 gp120}

The HF-TOC procedures were performed as described previously [8,22] with HIV-1 (JD) or $100 \mathrm{ng} / \mathrm{ml}$ of recombinant HIV-1 gp120 (IIIB). The medium was changed daily with fresh gp120 proteins. After 5 days, IFN $\gamma(500 \mathrm{IU} / \mathrm{ml})$ was added to the HF-TOC for 2 days as positive controls. Thymocytes were harvested at 7 days post-infection for analyses. Human fetal thymocytes were incubated with recombinant HIV-1 gp120 (IIIB or BaL), at 5 or 50 $\mathrm{ng} / \mathrm{ml}$ for various times and harvested for Western blot analyses. IFN $\gamma(200 \mathrm{IU} / \mathrm{ml})$ or SDF1a (1 ng/ml) were used as controls. 


\section{Western blot analysis}

Western blot analysis was performed with total thymocyte cell extracts as described [13]. The anti-p91 (STAT1) mAb, anti-P-STAT1-Tyr, anti-P-STAT1-Ser and anti-STAT2 polyclonal antibodies were used. Anti-tubulin mAb was used to monitor protein levels. Hela or $293 \mathrm{~T}$ cells treated with IFNa or IFN $\gamma(200 \mathrm{IU} / \mathrm{ml})$ were used as positive controls for the antiphospho-Stat1 antibodies.

\section{Reverse transcriptase polymerase chain reaction analysis}

RT-PCR analysis was performed using the Ambion RT-PCR kit (Ambion, Inc., Austin, TX, USA). The following oligonucleotides were used: human Mx1, cagcttcagaaggccatgctgcagctcctg (up primer) and ggggag agctgcaaggtggagegattctg (down primer); human Mx2, ggcgcgacacgcactctgtcaattctccagc (up) and ctgggatctgaaa gggaccggctcccagtcac (down); IP-10, ggaccacacagaggctg cetctcccatcac (up) and gggcccettgggaggatggcagtggaagtcc (down); and actin, atcatgtttgagaccttcaa (up) and catctc ttgctcgaagtcca (down). PCR reactions were run for 30 cycles of $94^{\circ} \mathrm{C}$ for $20 \mathrm{~s}, 60^{\circ} \mathrm{C}$ for 1 min, and $72^{\circ} \mathrm{C}$ for $2 \mathrm{~min}$. Similar results were observed with JD or NL4-3 infected samples.

\section{Microarray assay}

Total RNA from mock and HIV-infected (JD or NL4-3) Thy/Liv organs (12-14 dpi) was prepared using the TriReagent (Sigma, St Louis, MO, USA). Fluorescence-activated cell sorter analysis showed no significant thymocyte depletion in HIV-infected Thy/Liv organs in comparison with mock-infected samples, and HIV-infected Thy/Liv organs accumulated high levels of HIV-1 (p24). PolyA+ messenger RNA was isolated using the Fast-Track 2 kit (InvitroGene, Carlsbad, CA, USA). The cDNA microarray assay was carried as reported $[20,21,23]$. Isolated mRNA was reverse-transcribed with 5' Cy3 (HIV) or Cy5 (mock) labeled random 9-mers (Operon Technologies, Inc., Alameda, CA, USA). Reactions were incubated for $2 \mathrm{~h}$ at $37^{\circ} \mathrm{C}$ with $200 \mathrm{ng}$ polyA RNA, 200 units M-MLV reverse transcriptase (Life Technologies, Gaithersburg, MD, USA), 4 mM dithiothreitol, 1 unit RNase inhibitor (Ambion, Austin, TX, USA), $0.5 \mathrm{mM}$ dinucleotide triphosphates, and $2 \mu \mathrm{g}$ labeled 9-mers in $25 \mu \mathrm{l}$ volume with enzyme buffer supplied by the manufacturer. The reaction was terminated by incubation at $85^{\circ} \mathrm{C}$ for $5 \mathrm{~min}$. The probe was hybridized to the array, which was scanned in both $\mathrm{Cy} 3$ and $\mathrm{Cy} 5$ channels using Axon GenePix scanners (Foster City, CA, USA) with a $10 \mu \mathrm{m}$ resolution. The signal was converted into 16 bits-per-pixel resolution, yielding a 65 536 count dynamic range. Incyte GEMtools software (Incyte Pharmaceuticals, Inc., Palo Alto, CA, USA) was used for image analysis. The element signals were determined using a gridding and region detection algorithm. The area surrounding each element image was used to calculate a local background and was subtracted from the total element signal.

Background subtracted element signals were used to calculate Cy3 : Cy5 (if Cy3 > Cy5, thus positive) or $\mathrm{Cy} 5: \mathrm{Cy} 3$ (if $\mathrm{Cy} 5>\mathrm{Cy} 3$, thus negative) ratios. The average of the resulting total $\mathrm{Cy} 3$ and $\mathrm{Cy} 5$ signal gives a ratio that is used to balance or normalize the signals. In three independent experiments (two with JD and one with NL4-3), HIV-infected Thy/Liv organs were compared with mock-infected Thy/Liv organs. 


\section{Results}

Although high levels of HIV-1 were present at 2 weeks post-infection with HIV-1 (JD or NL4-3), no significant thymocyte depletion was detected (eight and data not shown). In addition to the induction of MHC I [13], HIV-1 infection of the Thy/Liv organ at 2 weeks post-infection leads to the profound suppression of the hematopoietic stem-progenitor cell activity in the thymus organ via indirect mechanisms $[18,19]$. We reasoned that HIV infection in the thymus led to the induction of host factors or signaling pathways that contribute to HIV replication and pathogenesis. To identify such 'pathogenic' host factors or pathways, we employed the cDNA microarray assay [20,21] to analyse the expression of 9183 host genes that were induced or suppressed by HIV infection in the thymus.

We designed our experiments as follows: to identify genes specifically induced or suppressed by HIV-1 infection with the cDNA microarray assay. First, the SCID-hu Thy/Liv model provided multiple Thy/Liv organs derived from the same donors in inbred mouse strains. No genetic variations thus existed between HIV and mock-infected samples in each experiment. Second, the 12 days post-infection timepoint was chosen because no significant thymocyte depletion was detected in HIV-infected Thy/Liv organs (eight and data not shown), so mRNA from the same cell types could be compared.

As a sensitivity control, known amounts of mRNA (2-2000 pg) from yeast-specific genes were mixed in the reactions with $200 \mathrm{ng}$ sample RNA, and were hybridized with yeast cDNA on the array slide; 2 pg RNA were reproducibly detected. A linear increase in signals was detected from 2 to $2000 \mathrm{pg}$ RNA. In addition, no signal spill-over was detected in wells adjacent to wells with high Cy3 or Cy5 signals (data not shown).

To demonstrate that different ratios of known RNA can be accurately quantitated, mRNA from different yeast-specific genes were added at indicated ratios to sample mRNA, labeled with $\mathrm{Cy} 3$ or Cy5, and hybridized on the cDNA arrays (Fig. 1a). A threefold difference (-threefold or +threefold) was consistently detected, and a linear correlation was observed from -25-fold (singlefold mRNA labeled with Cy3 and 25-fold mRNA with Cy5) to 25-fold (25-fold mRNA labeled with Cy3 and singlefold mRNA with Cy5) differences.

To monitor the quality of the HIV and mock mRNA samples, three human housekeeping genes (ribosomal S9, 23KDHBP, and a-tubulin) and three sets of human genes that are expressed in multiple tissues (complex targets) were arrayed in quadruplets (Fig. 1b). When the $\mathrm{Cy} 3$ (HIV)/Cy5 (mock) signals were analysed, the ribosomal S9 gene showed a -1.02 ( \pm 0.09 ) ratio, demonstrating 1.02-fold (almost identical levels) of S9 mRNA in mock-infected samples. The second control gene, 23KDHBP, showed a $-1.1( \pm 0.00)$ ratio. The a-tubulin gene showed a slight reduction in HIV-infected samples with a ratio of $-1.75( \pm 0.19)$. Three different sets of human genes (complex targets) showed -1.3 to $-1.5( \pm 0.2)$ ratios. In addition, the common thymocyte marker genes such as Thy-1, CD8 and TCR $\beta$ showed similar ratios $(-1.1,-1.2$ and -1.1 , respectively) between the two samples. The results indicated that mock and HIV-infected samples had generated high-quality RNA, and the major housekeeping genes were not altered by HIV infection. The microarray assay thus showed reproducible detection of $2 \mathrm{pg}$ RNA and threefold or higher differential (induction 
or suppression) expression. In three independent microarray assays with different donor tissues (two infected with JD and one with NL4-3), a similar number of genes were shown to be induced over 10 -fold $(0.04 \%)$, fivefold $(0.08 \%)$ or 2.5 -fold $(0.37 \%$, Fig. $1 \mathrm{c})$ in HIVinfected samples compared with either mock-infected samples.

When the data were analysed, 919 genes showed significant (> 1.5-fold) induction and 386 genes showed greater than 1.5 -fold suppression by HIV-1 infection (data not shown). When the genes with greater than threefold induction were analysed, $19 \mathrm{cDNA}$ showed (threefold to 15.7 -fold) induction by HIV infection (Table 1). Interestingly, 12 of the 19 induced genes encoded products involved in the IFN-Stat1 signaling pathway, similar to the IFN-regulated gene cluster recently reported [21]. For example, Mx1 and Mx2 genes, both IFN inducible, were induced 15.7-fold and fivefold, respectively. STAT1, IP-10, and MHCI (HLA) genes, which are implicated in or regulated by the IFN signaling pathway [24], were all induced by HIV infection. We confirmed the expression profiles of some of the induced genes by RTPCR (Fig. 2a, Table 1 and data not shown).

Consistent with the microarray assay, the MHC class I mRNA and proteins have previously been shown to be induced by HIV-1 infection $[1,13]$. As the activation of STAT1 could lead to the induction of the IFN-regulated gene cluster [24], we performed Western blot assays to analyse STAT1 expression. Both the STAT1 and STAT2 proteins were induced by HIV infection (Fig. 2b). Stat1 requires phosphorylation to be active [24]. To analyde the activation state of Stat1, antibodies specific for the active form of STAT1 (P-Ser727 or PTyr701) were used to detect phosphorylated STAT1 proteins in HIV-1-infected samples (Fig. 3). Both P-Ser727 and P-Tyr701 forms of Stat1 proteins were detected, suggesting that the STAT1 protein was also activated by HIV infection of the thymus.

Signaling through the HIV-1 co-receptor CXCR4 has recently been reported to activate STAT1 [25]. In addition, HIV-1 gp120 has been reported to induce CXCL10/IP-10 expression by astrocytes in vivo and in vitro, independent of IFN [26]. It is thus possible that the HIV-1 env protein gp120, which interacts with CD4 cells and co-receptors CXCR4 or CCR5 [27], may contribute to the induction of STAT1 and the IFN-regulated gene cluster. To test this possibility, we treated HF-TOC with recombinant HIV-1 gp120 (IIIB) proteins (Fig. 4a). As in the SCID-hu Thy/Liv mouse, HIV-1 infection led to the induction and activation of STAT1. Treatment of HF-TOC with HIV-1 gp120 proteins also led to the induction and activation of STAT1. We also incubated human fetal thymocytes with the recombinant gp120 proteins (IIIB and BaL). Expression of total STAT1 proteins was induced in cultured thymocytes with or without recombinant gp120 (Fig. 4b). Nonetheless, the activation of STAT1 proteins by phosphorylation at Tyr701 was only detected in thymocytes cultured with gp120 (IIIB or BaL), IFN $\gamma$ or SDF1. Therefore, HIV-1 gp120, via its interaction with its receptor (CD4) and co-receptors (CXCR4 or CCR5), may directly or indirectly contribute to the activation of the STAT1 signaling pathway.

\section{Discussion}

Destruction of the thymus organ by HIV-1 infection was probably caused by the induced expression of the host, as well as viral, pathogenic factors. Identification of the host factors 
or pathways involved will help our understanding of HIV pathogenesis in the thymus. Using the cDNA microarray assay (20, 21 and Fig. 1) and confirmed by RT-PCR or Western blot assays (Fig. 2), we showed that HIV-1 infection of the thymus organ led to the induction of a number of genes involved in the Stat1 signaling pathway (Table 1). We demonstrated that HIV-1 infection led to the induction and activation of the STAT1 protein (Fig. 3). We further showed that the incubation of HF-TOC or thymocytes with recombinant HIV-1 gp120 led to the induction and activation of the STAT1 protein (Fig. 4).

A recent microarray study with PBMC infected with HIV-1 [28] revealed the dysregulation of a number of human genes in response to HIV infection. However, no genes from the IFNregulated cluster were induced. This suggests that alteration of gene expression by HIV-1 in the thymus in vivo is different from that in activated PBMC in vitro. It has recently been reported that PBMC from HIV-infected patients or PBMC infected with HIV in vitro show increased activation of STAT1 and STAT5, although the level of total STAT proteins in PBMC is the same between HIV-positive and HIV-negative samples [29]. However, a different report [30] suggested that HIV-1 infection of PBMC in vitro or in vivo may reduce the expression of STAT5 and STAT1. Differences in sample treatment and experimental procedures may result in contradictory findings. Similar to PBMC isolated from HIVpositive patients, the majority of thymocytes in the Thy/Liv organ at 2 weeks post-infection are not directly infected by HIV-1 $[8,13]$, suggesting that indirect viral or host mediators are involved. The mechanism of HIV-1-induced STAT activation in the thymus and in PBMC in vivo may be similar, although thymocytes in vivo express very low levels of STAT1 (Fig. 2, Fig. 3, Fig. 4).

The mechanism and significance of HIV-1-induced activation of STAT1 and the IFNregulated gene cluster in the thymus are not clear. When levels of cell-associated major IFNa, $\beta$ or $\gamma$ proteins in the Thy/Liv organ were analysed by ELISA, no significant induction was detected in HIV-infected SCID-hu Thy/Liv thymocytes (data not shown). And no supernatants were available in the Thy/Liv organ model. Using a human fetal thymus organ culture model, it was recently reported that IFNa production was induced, predominantly from type 2 predendritic cells in HIV-infected thymus organs [31]. It is possible, therefore, that HIV infection or gp120 activated type 2 predendritic cells to produce IFNa, which may activate the Stat 1 pathway in thymocytes. The mRNA of IL-10, which has been reported to be induced in HIV-infected thymocytes and may contribute to MHC class I induction [13], was not significantly induced by the microarray assay (data not shown). As the expression of many cytokines is regulated at post-transcriptional levels, production of IL-10 protein may be regulated in HIV-infected thymus organs.

IFN-independent induction of 'IFN-responsive' RNA after human cytomegalovirus infection has previously been reported [32]. It is of interest that signaling through the HIV-1 co-receptor CXCR4 by SDF1 leads to the activation of STAT1 [25]. In addition, the expression of gp120 in astrocytes in vivo and in vitro leads to IFN-independent induction of IP-10 [26]. It is not clear whether signaling via CCR5 can also activate Stat 1 . As a coreceptor for HIV-1 entry, the interaction of CXCR4 or CCR5 with HIV-1 gp120 [27,33] may contribute to activation of the STAT1 pathway in human thymocytes (Fig. 4). In addition, peptides derived from HIV gp41 proteins have been shown to be structurally and 
functionally similar to type I IFN [34]. It is thus possible that HIV-encoded proteins such as gp120 and gp41 may be involved in the induction of STAT1 and the IFN-regulated gene cluster.

The effect of Stat1 induction and activation in thymocytes on HIV replication is not clear. Interestingly, a recent report [35] indicated that Stat1 activation was involved in the suppression of HIV-1 gene expression mediated by CD8 T cell antiviral factors. The induction and activation of Stat 1 may thus be a host cell response to resist infection. Activated STAT1 can also transduce signals affecting cell survival [36]. Thymocytes with elevated and activated STAT1 may become hypersensitive to IFN-like stimulation and to HIV-1 pathogenesis. In combination with other viral or host factors, Stat1 activation may contribute to thymocyte depletion. Further studies of HIV-1-induced activation of the STAT1 pathway in the thymus will help elucidate the mechanism of HIV-induced thymus destruction and define a novel target for modulating HIV pathogenesis.

\section{Acknowledgments}

The authors would like to dedicate this paper to the memory of Dr Eric D. Miller. They would also like to thank Drs Jenny Ting and Shannon Kenny for helpful discussions and reagents, Gregoriy Kovalev for help with the SCID-hu Thy/Liv infection, and Michael Townsend for technical assistance; the University of North Carolina at Chapel Hill Center for AIDS Research (CFAR, no. 9P30 AI50410), and the tissue culture and FACS facilities of the Lineberger Comprehensive Cancer Center at UNC, Chapel Hill for support.

Sponsorship: This work was supported by an NIH grant (AI41356 to L.S.). E.M. was supported by NIH (AI41356) and a fellowship from the American Foundation for AIDS Research (amfAR no 70520-28-RFI).

\section{References}

1. Courgnaud V, Laure F, Brossard A, Bignozzi C, Goudeau A, Barin F, Brechot C, et al. Frequent and early in utero HIV-1 infection. AIDS Res Hum Retroviruses. 1991; 7:337-341. [PubMed: 2064830]

2. Seemayer TA, Laroche AC, Russo P, Malebranche R, Arnoux E, Guerin J-M, et al. Precocious thymic involution manifested by epithelial injury in the acquired immune deficiency syndrome. Hum Pathol. 1984; 15:469-474. [PubMed: 6609873]

3. Kourtis AP, Ibegbu C, Nahmias AJ, Lee FK, Clark WS, Sawyer MK, Nesheim S. Early progression of disease in HIV-infected infants with thymus dysfunction. N Engl J Med. 1996; 335:1431-1436. [PubMed: 8875920]

4. McCune JM, Namikawa R, Kaneshima H, Shultz LD, Lieberman M, Weissman IL. The SCID-hu mouse: a model for the analysis of human hematolymphoid differentiation and function. Science. 1988; 241:1632-1639. [PubMed: 2971269]

5. Bonyhadi ML, Rabin L, Salimi S, Brown DA, Kosek J, McCune JM, Kaneshima H. HIV induces thymus depletion in vivo. Nature. 1993; 363:728-732. [PubMed: 8100043]

6. Aldrovandi GM, Feuer G, Gao L, Jamieson B, Kristeva M, Chen IS, Zack JA. The SCID-hu mouse as a model for HIV-1 infection. Nature. 1993; 363:732-736. [PubMed: 8515816]

7. Stanley SK, McCune JM, Kaneshima H, Justement JS, Sullivan M, Boone E, et al. Human immunodeficiency virus infection of the human thymus and disruption of the thymic microenvironment in the SCID-hu mouse. J Exp Med. 1993; 178:1151-1163. [PubMed: 8376927]

8. Su L, Kaneshima H, Bonyhadi M, Salimi S, Kraft D, Rabin L, McCune JM. HIV-1 induced thymocyte depletion is associated with indirect cytopathicity and infection of progenitor cells in vivo. Immunity. 1995; 2:25-36. [PubMed: 7600300]

9. Kaneshima H, Su L, Bonyhadi ML, Connor RI, Ho DD, McCune JM. Rapid-high, syncytiuminducing isolates of human immunodeficiency virus type 1 induce cytopathicity in the human thymus of the SCID-hu mouse. J Virol. 1994; 68:8188-8192. [PubMed: 7966610] 
10. Aldrovandi GM, Zack JA. Replication and pathogenicity of human immunodeficiency virus type 1 accessory gene mutants in SCID-hu mice. J Virol. 1996; 70:1505-1511. [PubMed: 8627668]

11. Jamieson BD, Aldrovandi GM, Planelles V, Jowett JB, Gao L, Bloch LM, et al. Requirement of human immunodeficiency virus type 1 nef for in vivo replication and pathogenicity. J Virol. 1994; 68:3478-3485. [PubMed: 8189487]

12. Jamieson BD, Uittenbogaart CH, Schmid I, Zack JA. High viral burden and rapid CD4+ cell depletion in human immunodeficiency virus type 1-infected SCID-hu mice suggest direct viral killing of thymocytes in vivo. J Virol. 1997; 71:8245-8253. [PubMed: 9343176]

13. Kovalev G, Duus K, Wang L, Lee R, Bonyhadi M, Ho D, McCune JM. Induction of MHC class I expression on immature thymocytes in HIV-1-infected SCID-hu Thy/Liv mice: evidence of indirect mechanisms. J Immunol. 1999; 162:7555-7562. [PubMed: 10358212]

14. Finkel TH, Banda NK. Indirect mechanism of HIV pathogenesis: how does HIV kill T cells? Curr Opin Immunol. 1994; 6:605-615. [PubMed: 7946050]

15. Finkel TH, Tudor-Williams G, Banda NK, Cotton MF, Curiel T, Monks C, et al. Apoptosis occurs predominantly in bystander cells and not in productively infected cells of HIV-and SIV-infected lymph nodes. Nat Med. 1995; 1:129-134. [PubMed: 7585008]

16. Rosenzweig M, Connole M, Forand-Barabasz A, Tremblay MP, Johnson RP, Lackner AA. Mechanisms associated with thymocyte apoptosis induced by simian immunodeficiency virus. $\mathrm{J}$ Immunol. 2000; 165:3461-3468. [PubMed: 10975867]

17. Bonyhadi M, Su L, Auten J, McCune J, Kaneshima H. Cytokine dysregulation in human fetal thymus organ culture and in SCID-hu THY/LIV mice following infection with HIV. Keystone Symposium: Control and manipulation of the immune response. J Cell Biochem. 1995; 255:S49.

18. Jenkins M, Hanley MB, Moreno MB, Wieder E, McCune JM. Human immunodeficiency virus-1 infection interrupts thymopoiesis and multilineage hematopoiesis in vivo. Blood. 1998; 91:26722678. [PubMed: 9531575]

19. Koka PS, Fraser JK, Bryson Y, Bristol GC, Aldrovandi GM, Daar ES, Zack JA. Human immunodeficiency virus inhibits multilineage hematopoiesis in vivo. J Virol. 1998; 72:5121-5127. [PubMed: 9573283]

20. DeRisi JL, Iyer VR, Brown PO. Exploring the metabolic and genetic control of gene expression on a genomic scale. Science. 1997; 278:680-686. [PubMed: 9381177]

21. Perou CM, Jeffrey SS, van de Rijn M, Rees CA, Eisen MB, Ross DT, et al. Distinctive gene expression patterns in human mammary epithelial cells and breast cancers. Proc Natl Acad Sci U S A. 1999; 96:9212-9217. [PubMed: 10430922]

22. Su L, Kaneshima H, Bonyhadi ML, Lee R, Auten J, Wolf A, et al. Identification of HIV-1 determinants for replication in vivo. Virology. 1997; 227:45-52. [PubMed: 9007057]

23. Feske S, Giltnane J, Dolmetsch R, Staudt LM, Rao A. Gene regulation mediated by calcium signals in T lymphocytes. Nat Immunol. 2001; 2:316-324. [PubMed: 11276202]

24. Darnell JE Jr. STATs and gene regulation. Science. 1997; 277:1630-1635. [PubMed: 9287210]

25. Vila-Coro AJ, Rodriguez-Frade JM, Martin De Ana A, Moreno-Ortiz MC, Martinez AC, Mellado M. The chemokine SDF-1alpha triggers CXCR4 receptor dimerization and activates the JAK/ STAT pathway. FASEB J. 1999; 13:1699-1710. [PubMed: 10506573]

26. Asensio VC, Maier J, Milner R, Boztug K, Kincaid C, Moulard M, et al. Interferon-independent, human immunodeficiency virus type 1 gp120-mediated induction of CXCL10/IP-10 gene expression by astrocytes in vivo and in vitro. J Virol. 2001; 75:7067-7077. [PubMed: 11435587]

27. Berger EA, Murphy PM, Farber JM. Chemokine receptors as HIV-1 coreceptors: roles in viral entry, tropism, and disease. Annu Rev Immunol. 1999; 17:657-700. [PubMed: 10358771]

28. Geiss GK, Bumgarner RE, An MC, Agy MB, van 't Wout AB, Hammersmark E, et al. Large-scale monitoring of host cell gene expression during HIV-1 infection using cDNA microarrays. Virology. 2000; 266:8-16. [PubMed: 10612655]

29. Bovolenta C, Camorali L, Lorini AL, Ghezzi S, Vicenzi E, Lazzarin A, Poli G. Constitutive activation of STATs upon in vivo human immunodeficiency virus infection. Blood. 1999; 94:4202-4209. [PubMed: 10590065] 
30. Pericle F, Pinto LA, Hicks S, Kirken R, Sconocchia G, Rusnak J, et al. HIV-1 infection induces a selective reduction in STAT5 protein expression. J Immunol. 1998; 160:28-31. [PubMed: 9551952]

31. Keir ME, Stoddart CA, Linquist-Stepps V, Moreno ME, McCune JM. IFN-alpha secretion by type 2 predendritic cells up-regulates MHC class I in the HIV-1-infected thymus. J Immunol. 2002; 168:325-331. [PubMed: 11751977]

32. Zhu H, Cong JP, Shenk T. Use of differential display analysis to assess the effect of human cytomegalovirus infection on the accumulation of cellular RNAs: induction of interferon-responsive RNAs. Proc Natl Acad Sci U S A. 1997; 94:13985-13990. [PubMed: 9391139]

33. Su SB, Ueda H, Howard OM, Grimm M, Gong W, Ruscetti F, et al. Inhibition of the expression and function of chemokine receptors on human CD4+ leukocytes by HIV-1 envelope protein gp120. Chem Immunol. 1999; 72:141-160. [PubMed: 10550935]

34. Chen YH, Dierich MP. A common immunological epitope existing between HIV-1 gp41 and human interferon-alpha and -beta. Immunobiology. 1998; 198:333-342. [PubMed: 9562860]

35. Chang TL, Mosoian A, Pine R, Klotman ME, Moore JP. A soluble factor(s) secreted from CD8(+) T lymphocytes inhibits human immunodeficiency virus type 1 replication through STAT1 activation. J Virol. 2002; 76:569-581. [PubMed: 11752148]

36. Chin YE, Kitagawa M, Kuida K, Flavell RA, Fu XY. Activation of the STAT signaling pathway can cause expression of caspase 1 and apoptosis. Mol Cell Biol. 1997; 17:5328-5337. [PubMed: 9271410] 
(a)

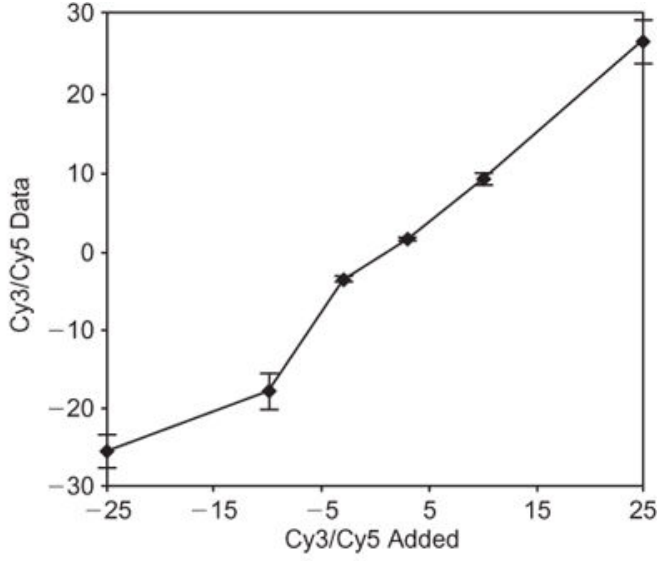

(b)

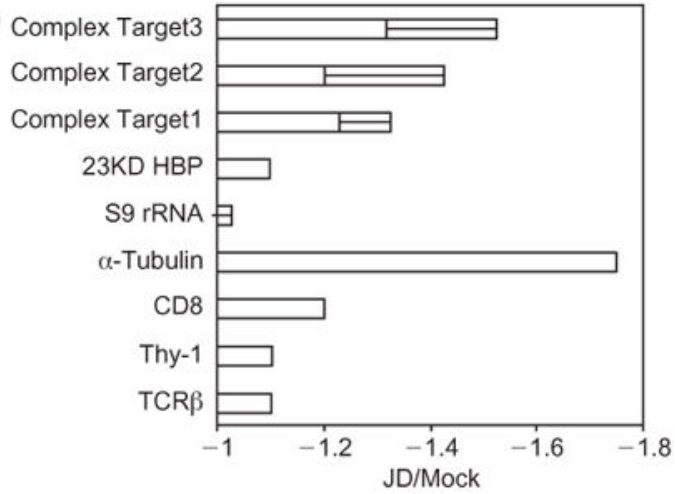

(c)

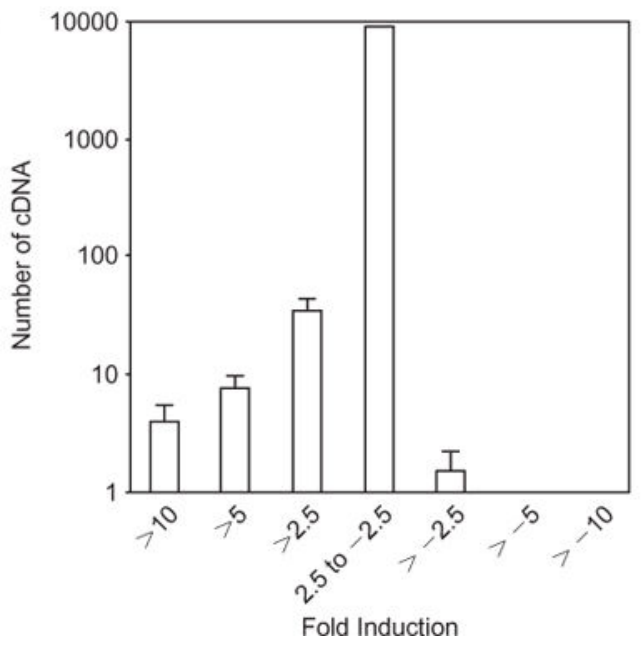

Fig. 1. Microarray assay of gene expression in HIV-1-infected Thy/Liv organs

(a) Quantitative measurement of differential gene expression. RNA from six different yeastspecific genes was mixed with the HIV or mock-infected RNA samples in the microarray assay. RNA from each yeast gene was added at a known ratio (3, 10 or 25) in the Cy3 (HIV) or Cy5 (mock) sample (Cy3/Cy5 added). The six yeast genes were arrayed on the slides in quadruplets. Positive values indicate threefold, 10-fold or 25-fold more RNA were used in the Cy3 (HIV) sample. Negative values $(-3,-10$ or -25$)$ indicate that threefold, 10 -fold or 25 -fold more RNA were included in the Cy5 (mock) sample. An equal amount of RNA in 
Cy3 and Cy5 samples is indicated as $1(+1$ or -1$)$. The $\mathrm{X}$ axis shows the added ratios and the $\mathrm{Y}$ axis indicates $\mathrm{Cy} 3 / \mathrm{Cy} 5$ readout data from the assay. Error bars indicate standard deviations. (b) RNA sample and reaction control. RNA samples from HIV $\mathrm{JD}_{\mathrm{JD}}$ (Cy3 labeled) or mock (Cy5 labeled) infected severe combined immunodeficient (SCID)-hu Thy/Liv mice were analysed using the microarray assay. Relative expression levels of $23000 \mathrm{M}_{\mathrm{r}}$ highly basic protein (HBP), ribosomal subunit S9 (S9 rRNA) and a-tubulin were quantified in quadruplets. Three sets of human genes known to express in multiple tissues (complex target 1-3) were also included as controls. Positive values (Cy3 signal/Cy5 signal) indicate more RNA in the Cy3 (HIV) sample, thus induction of expression by HIV. Negative values (Cy5 signal/Cy3 signal) indicate more RNA in the Cy5 (mock) sample, thus suppressed expression by HIV. Error bars indicate standard deviations from the quadruplet arrays. The relative expression of three T cell markers (TCR $\beta, \mathrm{CD} 8$ and Thy-1) from the microarray assay was also shown. Comparison of HIV (JD or NL4-3) and mock infected samples from different donor tissues showed similar results. (c) Alteration of gene expression in SCID-hu Thy/Liv mice after HIV-1 infection. A small number $(<0.2 \%)$ of genes were induced (> threefold) in HIV-1-infected Thy/Liv organs over mock infected samples. The majority of genes were in the +2.5 to -2.5 range $(99.5 \%)$. Error bars indicate standard deviations from three experiments (two with JD and one with NL4-3) with different donor tissues. 
(a)

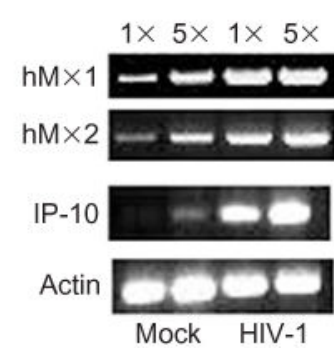

(b)

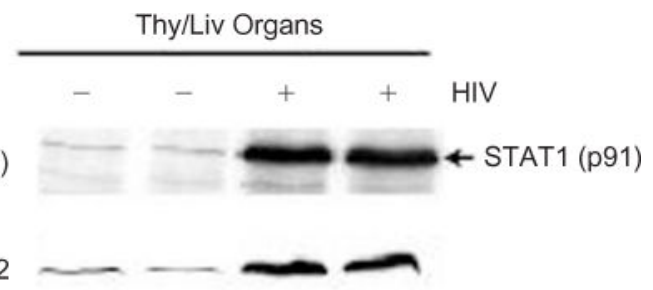

anti- $\beta$-Tubulin

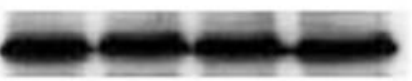

Fig. 2. Induction of the IFN-induced gene cluster and of the signal transducer and activator of transcription 1 protein in HIV-1-infected Thy/Liv organs

(a) Confirmation of gene induction by reverse trancriptase-polymerase chain reaction (RTPCR). Severe combined immunodeficient (SCID)-hu Thy/Liv mice infected with mock (-) or with HIV-JD (+) were harvested at 2 weeks post-infection. Total thymocyte RNA from mock or HIV-infected Thy/Liv organs were analysed by RT-PCR with primers specific for the selected genes. $1 \times$ and $5 \times$ indicate $25 \mathrm{ng}$ and $125 \mathrm{ng}$ of total RNA used in the RT-PCR reaction. Expression of actin was used to monitor cellular RNA. No significant thymocyte depletion was detected in mock or HIV-infected samples. Two independent experiments with different donor tissues were performed with similar results. (b) Induction of signal transducer and activator of transcription (STAT)1 and STAT2. SCID-hu Thy/Liv mice infected with mock (-) or with HIV-JD (+) were harvested at 2 weeks post-infection. Total thymocyte proteins $(50 \mu \mathrm{g})$ from two representative mock or two HIV-infected Thy/Liv organs were analysed by Western blot with the anti-p91 (STAT1) monoclonal antibody or anti-STAT2 polyclonal antibody. Anti- $\beta$-tubulin antibody was used to monitor cellular proteins. No significant thymocyte depletion was detected in mock or HIV-infected samples. Four independent experiments (infected with JD or NL4-3) with different donor tissues were performed with similar results. 


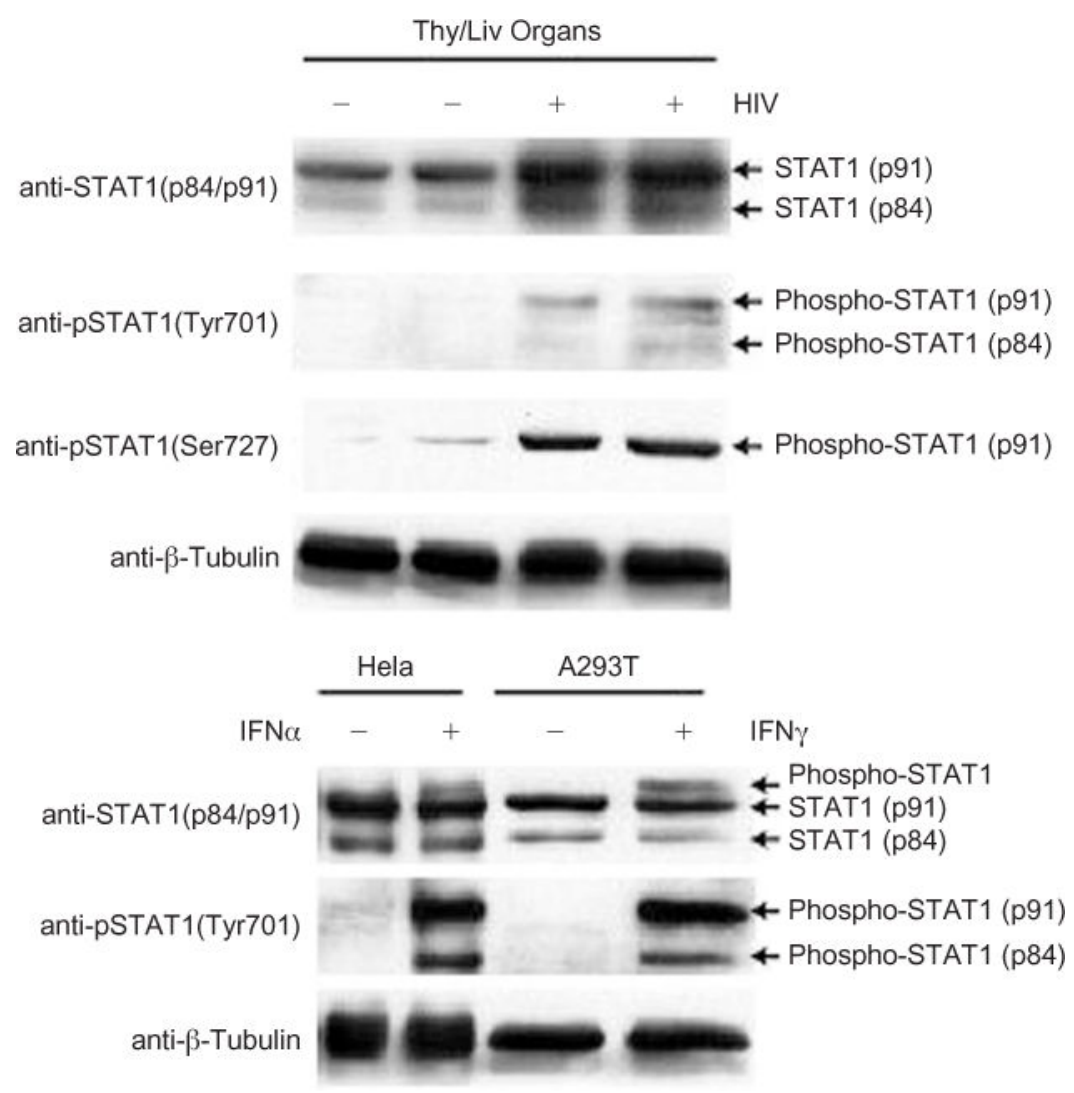

Fig. 3. Activation of the signal transducer and activator of transcription 1 protein in HIV-1infected Thy/Liv organs

Total thymocyte proteins were blotted with polyclonal anti-signal transducer and activator of transcription (STAT)1 (p84/p91) antibody or with antibodies specifically reactive with STAT1 phosphorylated at Ser727 (pSTAT1-Ser) or Tyr701 (pSTAT1-Tyr). Anti-pSTAT1 (Y701) detects both forms of phospho-STAT1 (p91 and p84) and anti-pSTAT1 (Ser727) is specific to phospho-STAT1-p91. Two representative mock or HIV-(JD)-infected Thy/Liv samples were presented. Proteins from IFNa-treated Hela or IFN $\gamma$-treated 293T cells were used as positive control for the anti-P-STAT1 (Y701) antibody. Three independent experiments (infected with JD or NL4-3) with different donor tissues were performed with similar results. 


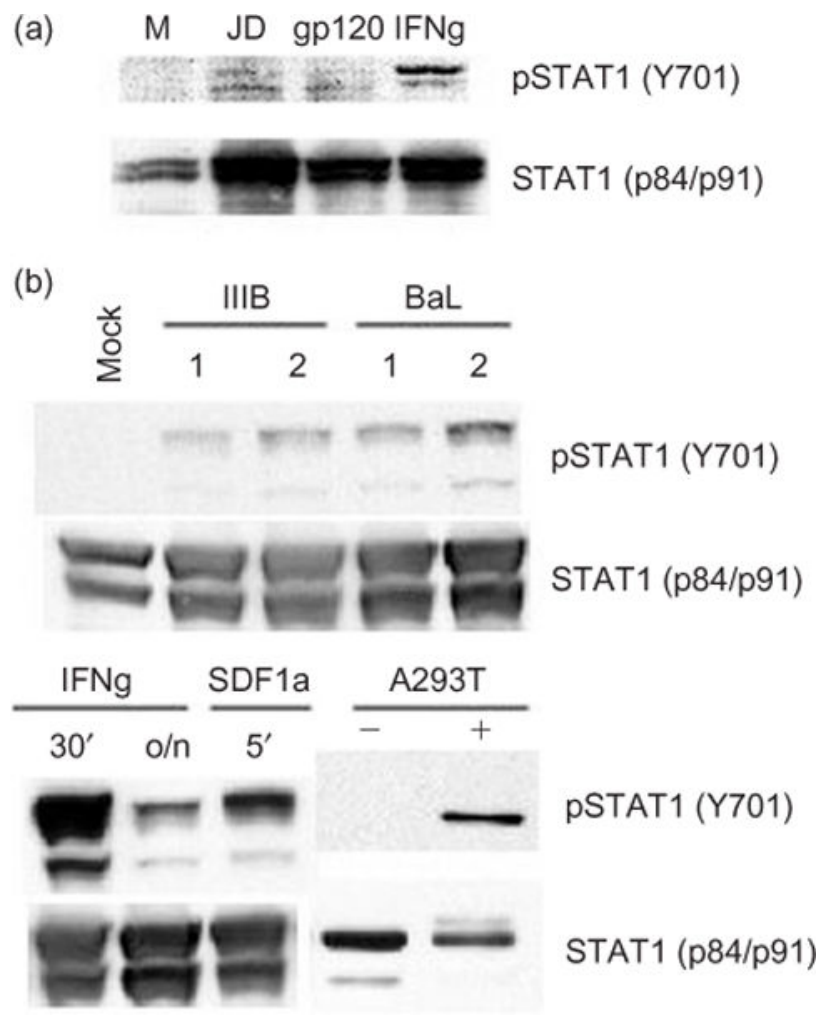

Fig. 4. Activation of signal transducer and activator of transcription 1 in human fetal thymus organ culture or thymocytes by HIV infection or recombinant HIV-1 gp120

(a) Induction and activation of signal transducer and activator of transcription (STAT)1 in human fetal thymus organ culture (HF-TOC) by HIV-1 infection and recombinant gp120. HF-TOC infected with mock (M) or HIV-JD, or treated with recombinant gp120 (IIIB, 100 $\mathrm{ng} / \mathrm{ml}$ ) or IFN $\gamma$ were analysed for STAT1 protein expression (STAT1 p84/p91) and activation (pSTAT1Y701) as described above. NL4-3 infection also led to STAT1 activation (not shown). (b) Activation of STAT1 in human thymocytes by recombinant gp120. Human fetal thymocytes were cultured with medium (mock), with $5 \mathrm{ng} / \mathrm{ml}$ (lanes 1) or $50 \mathrm{ng} / \mathrm{ml}$ (lanes 2) of recombinant HIV-1 gp120 (IIIB or BaL) preparation. Thymocytes were harvested at $12 \mathrm{~h}(\mathrm{o} / \mathrm{n})$ post culture and analysed for total STAT1 proteins $(\mathrm{p} 84 / \mathrm{p} 91)$ and Tyr701 phosphorylated STAT1 (Y701). Human thymocytes treated with IFN $\gamma$ or SDF1a were used as controls. 


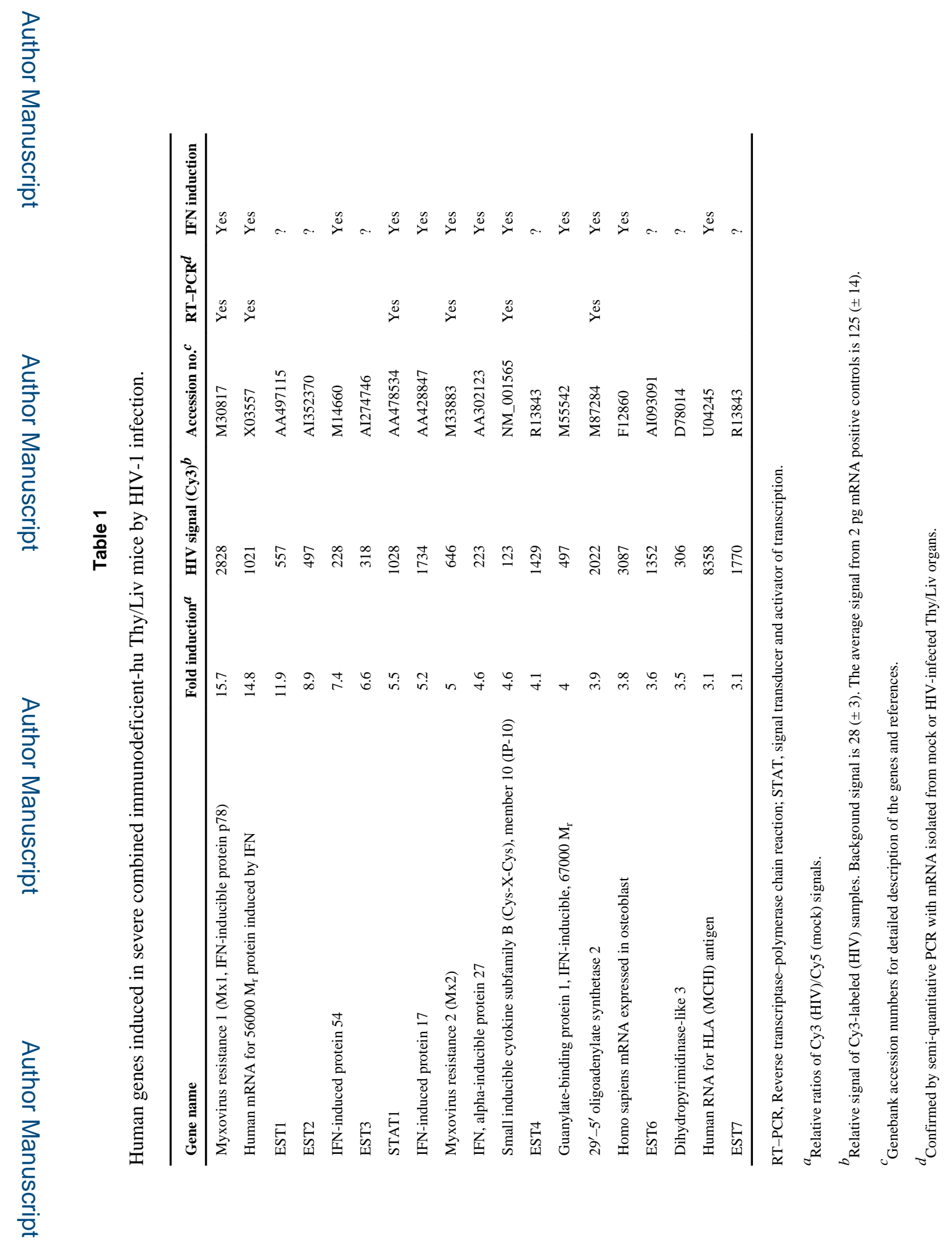

AIDS. Author manuscript; available in PMC 2015 April 30. 